\title{
DISSOCIATION OF ELECTRICAL AND MECHANICAL ACTIVITY IN THE FROG'S SCIATIC-SARTORIUS MUSCLE PREPARATION CAUSED BY PROLONGED IMMERSION IN RINGER SOLUTION
}

\author{
Tuyoshi KIKU-IRI* \\ Department of Physiology, Sapporo Medical College, Sapporo, Japan
}

\begin{abstract}
Recent physiological studies on muscles have shown that the excitation and contraction phenomena can be easily dissociated by the treatments with hypertonicity ${ }^{1,2)}$ chemical substances ${ }^{3,4)}$ and others ${ }^{5,6,7)}$, and suggested the existence of some processes linking excitation of the muscle membrane and contraction of muscle fibre (E-C coupling processes) ${ }^{8}$.

It has been demonstrated that the failure of end-plate transmission occurs in both fatigued ${ }^{9)}$ and denervated ${ }^{10,11)}$ nerve-muscle preparations. Recently MERTON $(1954)^{5)}$, SERAYDARIAN $\left.(1961)^{6}\right)$ and MASHima et al. (1962) have shown that the E-C coupling processes diminished by muscular fatigue which was independent of the failure of end-plate transmission. Based on these reports, there may arise some questions to investigate whether the E-C coupling processes are influenced by the prolonged immersion in Ringer solution and whether the immersion causes the same kind of effects as the denervation on the excitability and contractility of muscle.

The present paper deals with the relationship between excitation and contraction in the isolated sciatic-sartorius preparation of frog immersed in Ringer solution for various periods up to 24 hours.
\end{abstract}

\section{MATERIALS AND METHODS}

1. Materials. In the present experiments, the sciatic-sartorius preparations of frog (Rana japonica and $R$. nigromaculata) were used. The preparations were immersed in about $300 \mathrm{ml}$ of Ringer solution for desired periods up to 24 hours at room temperature $\left(17-20^{\circ} \mathrm{C}\right)$. The control experiments were carried out in the contralateral preparations of the same frog immersed in Ringer solution for 2 hours. The bathing solution was not changed during the experiment.

2. Recording of mechanical phenomena. The muscle was mounted vertically in a

Received for publication July 13, 1962.

* 菊入 剛 
cylindrical chamber. The pelvic end of the muscle preparation was fixed with a glass hook at the bottom, and the tibial end was connected with the isotonic lever loaded with $1 \mathrm{~g}$. weight. The nerve was placed on a shallow tray which extruded horizontally at the upper end of the chamber. The tray containing the nerve trunk was electrically separated from the chamber containing the muscle with colophony bee wax. The muscle preparation was directly and transversely stimulated with the silver-silver chloride plate electrodes by $1.0 \mathrm{v} / \mathrm{cm}$ rectangular pulses of $0.5 \mathrm{msec}$ duration. The indirect stimulations were applied with $0.5 \mathrm{v} / \mathrm{cm}$ rectangular pulses of $0.5 \mathrm{msec}$ duration.

During the experiments the bathing solution was continuously stirred by small air bubbles coming out of a capillary tube set on the bottom of the chamber. The mechanical responses to electrical stimulations were recorded kymographically.

3. Recording of electrical phenomena. The action potentials of the nerve trunks were externally recorded by the method of Tasaki ${ }^{12}$, and the action potentials of the muscle fibres elicited by direct stimulations were intracellulary recorded by the method of Macfarlene and Mears ${ }^{13}$. The action potentials of the whole muscles elicited by indirect stimulations were externally recorded with the modification of STÄMFLI's method ${ }^{14)}$. In the present method the nerve-free part of the sartorius muscle was electrically separated from the nervous part by the isotonic sucrose gap. The recording electrodes were placed in each part of the chamber interposed by the gap.

4. Solutions. The normal Ringer solution was prepared with $112 \mathrm{mM} \mathrm{NaCl}, 2.0 \mathrm{mM}$ $\mathrm{KH}_{2} \mathrm{PO}_{4}$ and $1.3 \mathrm{mM} \mathrm{CaCl}$. The $\mathrm{pH}$ of the solution was adjusted to $7.0-7.2$ by addition of $\mathrm{M} / 8 \mathrm{NaHCO}_{3}$.

Caffeine and acetylcholine chloride (Ach) were respectively dissolved in the normal Ringer solution and diluted to the desired concentrations.

\section{RESULTS}

I. Changes in the mechanical responses. The mechanical responses evoked by electrical stimulations in the surviving muscles gradually decreased with the lapse of time after immersing the preparations in Ringer solution (FIG. 1), while no change in the twitch responses in the control muscle was observed. The reduced twitch responses in the directly stimulated muscle were parallel with those induced by indirect stimulations until 8 hours after immersion. Thereafter the reduction in the latter became more prominent.

At 24 hours after immersion the twitch responses evoked by indirect stimulations completely disappeared, whereas the directly stimulated muscle still induced the twitch responses although the twitch height was below 10\% of the control (FIG. 1).

These phenomena depended on the temperature of the bathing solution in which the preparations were immersed. The higher the temperature, the more quickly decreased the heights of twitch responses.

II. Action potentials of the nerve trunks of the preparations. Action potentials of the nerve elicited by electrical stimulations on the nerve trunks of the preparations were recorded at 2 and 24 hours after immersion. The feature of the action potentials in the preparation surviving for 24 hours was quite similar to that in the control (FIG. 2). 


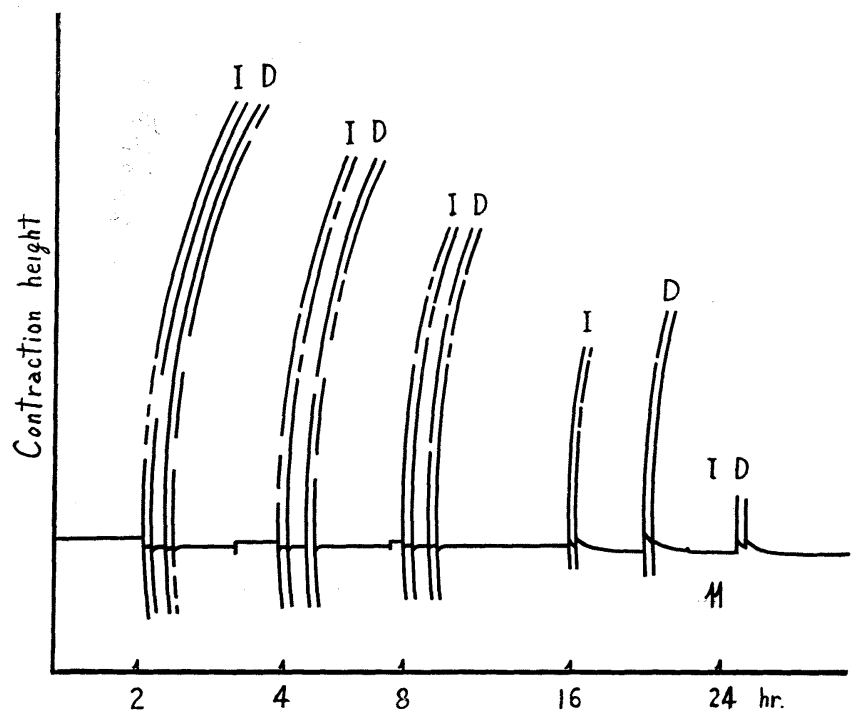

FIG. 1. Changes in mechanical responses of frog's sartorius muscle to indirect and direct stimulations at various time after immersing in Ringer solution. I and D represent the mechanical responses (twitch) to indirect and direct stimulations respectively. The slight fluctuations on the base line of figure are artifacts on recording. Note the negative response to indirect stimulation at 24 hours after immersion of muscle in Ringer solution.

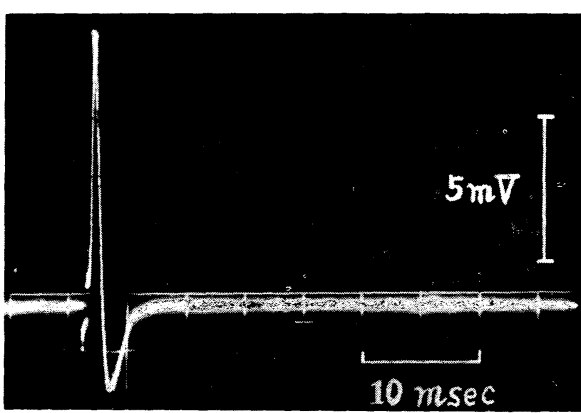

(a)

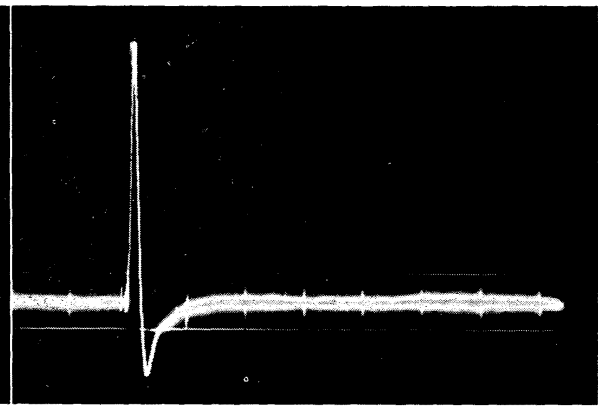

(b)

FIG. 2. Action potentials of the same nerve trunks of the nerve-muscle preparation immersed in Ringer solution for 2 (a) and 24 (b) hours.

III. Electrical properties of single muscle fibres of the nerve-muscle preparation. The resting and action potentials elicited by direct stimulations on single muscle fibres were recorded in four pairs of nerve-muscle preparations. The recordings were randomly carried out on five different single muscle fibres from a preparation. As shown in FIG. 3 and TABLE 1, the magnitudes of resting and action potentials of the fibres immersed in Ringer solution for 24 hours were quite similar to those of the control. 


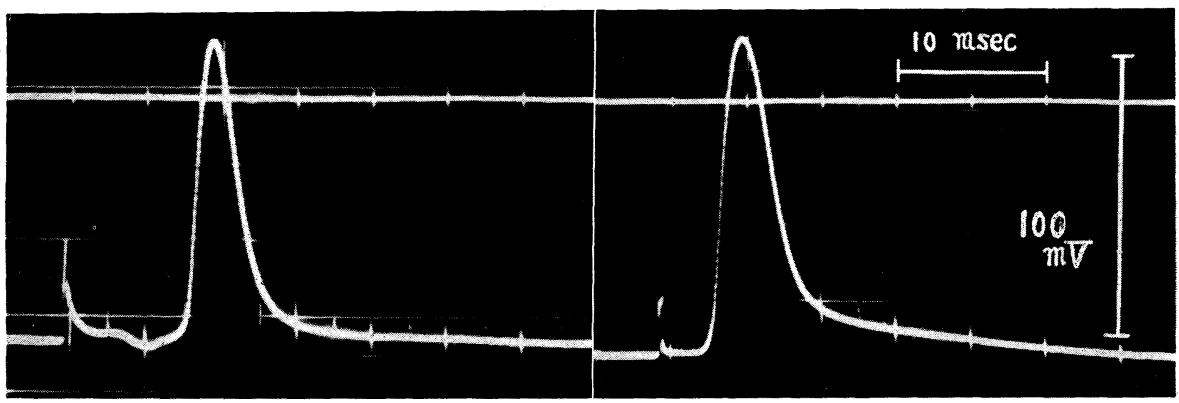

(a)

(b)

FIG. 3. The resting and action potentials of single muscle fibres from frog's sartorius immersed in Ringer solution for 2 (a) and 24 (b) hours. The former is the control of the latter (b).

\section{TABLE 1}

The transmembrane resting and action potentials measured in single muscle fibres of frog's sartorius preparation immersed in Ringer solution for 2 and 24 hours. In each case the measurements were performed on a pair of muscles.

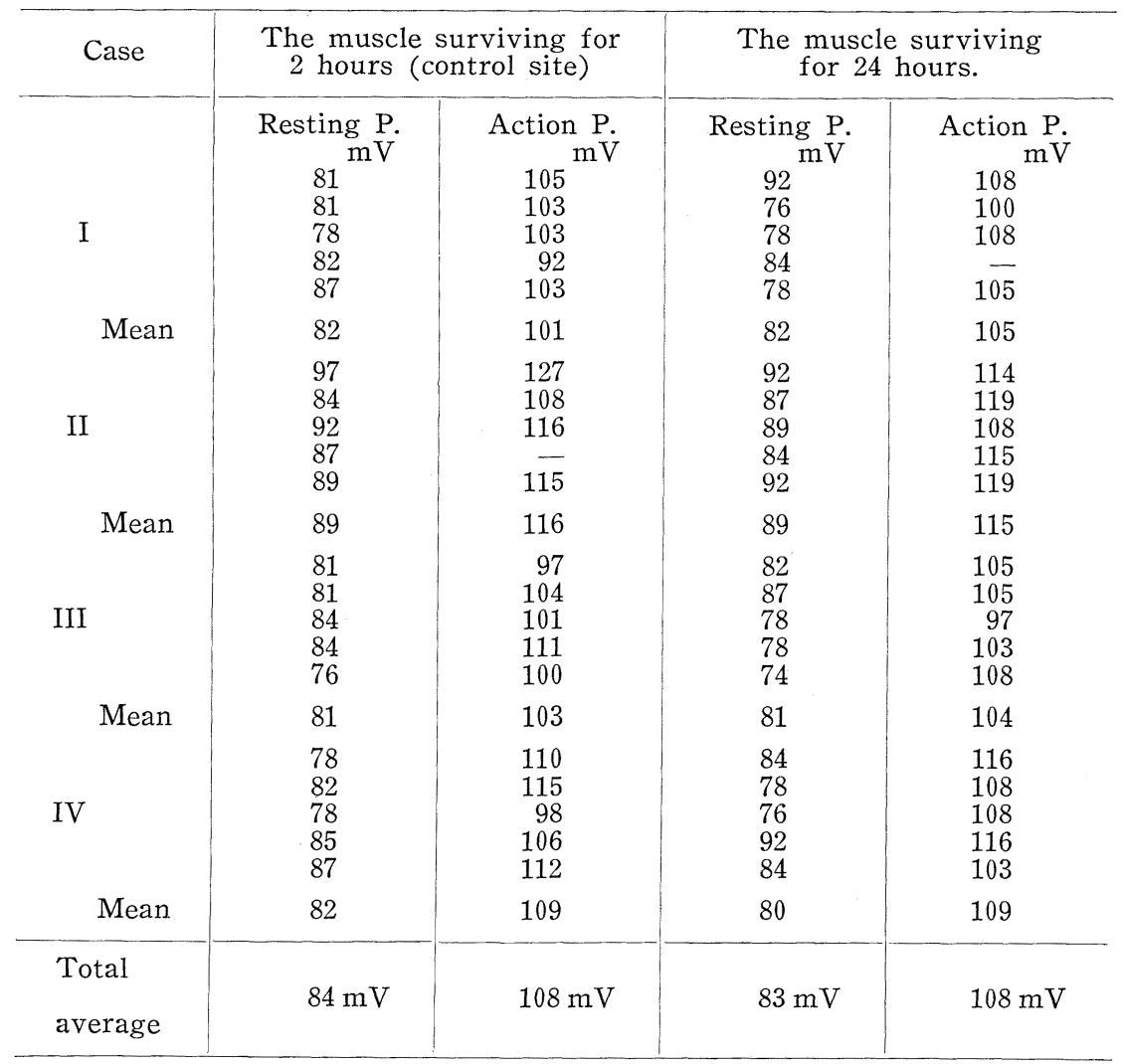


IV. Action potentials of the whole muscle preparations elicited by indirect stimulations. Although the twitch responses in the nerve-muscle preparation evoked by direct or indirect stimulations gradually decreased (FIG. 1), the action potentials of the whole muscles stimulated indirectly at 8 hours after immersion were almost similar to those of the control (FIG. 4).

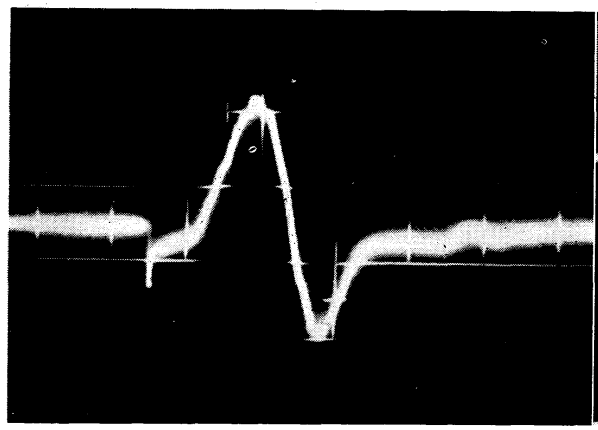

(a)

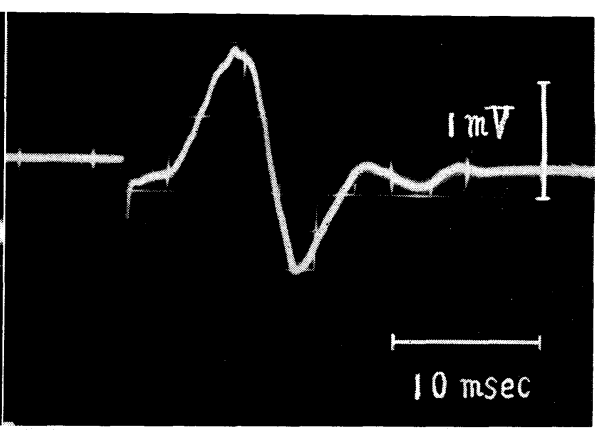

(b)

FIG. 4. Action potentials obtained from the whole muscle elicited by indirect stimulations at 2 (a) and 8 (b) hours after immersion in Ringer solution.

$V$. Caffeine-induced contracture of the nerve-muscle preparation. The irreversible contracture of the muscle was induced by caffeine over $5.0 \mathrm{mM}$ in concentration. When $4.0 \mathrm{mM}$ caffeine was applied to muscle, the size of the second contracture was much smaller than the first. $4.5 \mathrm{mM}$ caffeine reversibly in-

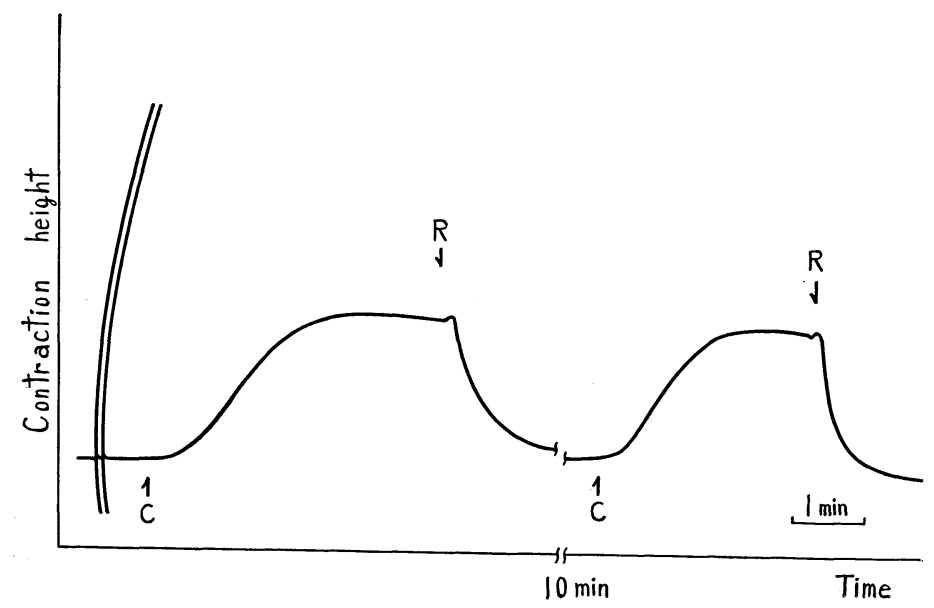

FIG. 5. Caffeine contracture and the reversibility in the frog's sartorius muscle immersed in Ringer solution for 2 hours (the control of FIG. 6). The upstrokes show the twitch responses to direct stimulations. The mark $\mathrm{C} 1$ shows the time when the $4.5 \mathrm{mM}$ caffeine was applied. Arrow mark $\mathrm{R} \downarrow$ shows the time when the caffeine was washed out of the contracted muscle with Ringer solution. 
duced the second contracture as high as the first one (FIG. 5). FIG. 6 represents one of the contractures induced by $4.5 \mathrm{mM}$ caffeine in the muscle at 24 hours after immersion in Ringer solution. In this case, the size of the contracture was quite similar to that of the control muscle (FIG. 5), whereas the twitch responses in the same muscle stimulated directly decreased remarkably. VI. Sensitivity to Ach of the nerve-muscle preparation. In control muscle $10^{-6}$ to

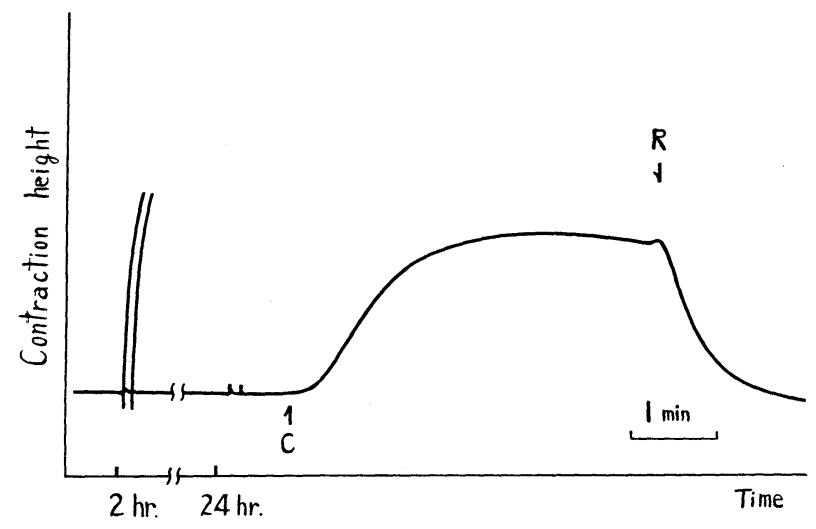

FIG. 6. Caffeine contracture in the frog's sartorius muscle at 24 hours after immersion in Ringer solution. Large and small upstrokes indicate the twitch responses evoked by direct stimulations at 2 and 24 hours after the immersion. The mark $\mathrm{C} 1$ shows the time when $4.5 \mathrm{mM}$ caffeine was applied. Arrow mark $\mathrm{R} \downarrow$ shows the time when the caffeine was washed out of the contracted muscle with Ringer solution.

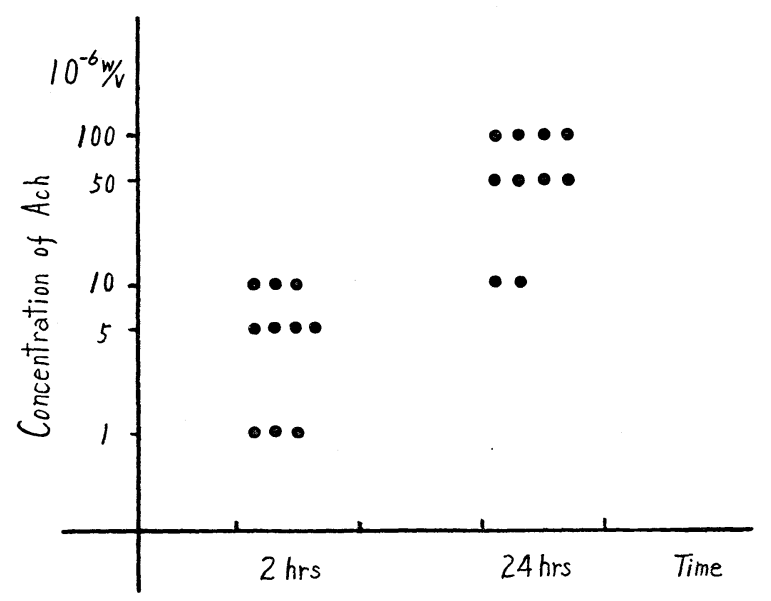

FIG. 7. The threshold concentrations of Ach to induce the contractures in the muscles immersed in Ringer solution for 2 and 24 hours. Each dot represents a surviving nerve-muscle preparation in which contracture was induced by Ach. 
$10^{-5} \mathrm{w} / \mathrm{v}$ Ach induced the transient contracture. In the muscle surviving for 24 hours in Ringer solution, however, no contracture was produced by Ach below $10^{-5} \mathrm{w} / \mathrm{v}$ in concentration (FIG. 7). In other words, the sensitivity to Ach of the preparation was lowered by immersion in Ringer solution.

\section{DISCUSSION}

In most experiments the twitch responses of frog's muscle evoked by direct electrical stimulations gradually decreased with the lapse of time after immersing the nerve-muscle preparations in Ringer solution at room temperature $\left(17-20^{\circ} \mathrm{C}\right)$. In spite of the reduction in twitch responses of the muscle, the resting and action potentials recorded from single muscle fibres were not changed (FIG. 1, 3 and TABLE 1). Furthermore, from the results that the size of caffeine contractures was quite similar to that in the control muscles (FIG. 6 ), it is considered that the contractile elements of the muscle are intact. The twitch responses evoked by indirect stimulations also decreased gradually with the lapse of time after immersion, while the action potentials from the nerve trunks and directly stimulated single muscle fibres did not change during this period (FIG. 1, 2, 3 and Table 1). Nicholls $(1959)^{15}$ ) reported that the resting and action potentials in the denervated skeletal muscle of frog remained unchanged for 40 days after the section of motor nerve. Using denervated frog's muscle, BIRKS and KATZ (1960) ${ }^{11)}$ observed that the end-plate transmission was deprived in relatively earlier stage. In the present study, however, the muscle immersed in Ringer solution up to 8 hours showed a similar decrease in the twitch responses both to direct and to indirect stimulations. This result could not be explained merely by the failure of end-plate transmission, because the size of twitch responses of directly stimulated whole muscle should be the same as that of the control if the reduction of twitch responses was due to the transmission failure alone. For explanation of this result, two possibilities were considered: i) E-C coupling processes can be impaired, while the endplate transmission is intact. ii) E-C coupling processes and end-plate transmission can be impaired respectively at the same period.

As described above, the twitch responses of the preparation to both types of stimulation gradually decreased in the same manner up to 8 hours after immersion (FIG. 1). On the other hand, the size of action potentials elicited by indirect stimulations on the whole muscle surviving for 8 hours was almost similar to that of the control muscle (FIG. 4). These results may exclude the second possibility, because the action potential of whole muscle recorded externally depends on numbers of exciting muscle fibres ${ }^{7,19}$. Therefore, it may be concluded that the reduction in size of twitch responses is due to the gradual impairment of the E-C coupling processes until 8 hours after immersion.

The prominent diminution in size of twitch responses evoked by indirect 
stimulations on the preparation immersed in Ringer solution for more than 8 hours may be considered as a result of the failure of end-plate transmission, because the twitch responses of the muscle to direct stimulations were less affected by the prolonged immersion than the responses to indirect ones. At 24 hours of immersion, the twitch responses of the surviving muscle to direct stimulations still remain, while those to indirect stimulations disappear (FIG. 1). Therefore, it may be concluded that the impairment of the E-C coupling processes starts in early stage of immersion and then the end-plate transmission begins to fail after 8 hours of immersion. And after the complete failure of end-plate transmission at 24 hours of immersion, the failure of the $\mathrm{E}-\mathrm{C}$ coupling processes still remains incomplete.

The fact that the decrease of twitch responses in the surviving muscle to electrical stimulations depends on the temperature of surrounding medium may suggest the presence of a relation between the impairment of the $\mathrm{E}-\mathrm{C}$ coupling processes and metabolic changes in muscle ${ }^{16)}$.

The present result that the failure of end-plate transmission was also influenced by the temperature of bathing solution coincides with the results obtained in the denervated muscle. As far as the influence of temperature upon the failure of end-plate transmission and changes in the electrical properties of the muscle membrane are concerned, the nerve-muscle preparation immersed in Ringer solution seems to have similar properties to those of denervated muscle. In the surviving muscle, however, the threshold concentration of Ach for causing detectable contracture was about 10 times higher than that in the control (FIG. 7), whereas the supersensitivity to Ach has been well known in the denervated skeletal muscle ${ }^{17,18)}$. From the results it may be considered that the impairment of the E-C coupling processes in surviving muscle did not result from denervation, but was solely due to a chemical or a physicochemical change in muscle caused by the prolonged immersion in Ringer solution.

\section{SUMMARY}

The effects of the immersion of nerve-muscle preparations of frog in Ringer solution on the excitation and contraction phenomena were followed up to 24 hours.

1. The twitch responses of the muscle to indirect and direct electrical stimulations decreased to quite similar degree until 8 hours after immersion. However, it was observed that the twitch responses to indirect stimulations reduced more prominently after 8 hours.

2. Until at least 8 hours after immersion, no changes in action potentials of the whole muscles elicited by indirect stimulations were observed.

3. Not only the action potentials of the nerve trunks, but also the resting 
and action potentials of the directly stimulated muscle fibres were quite similar to those in the control even at 24 hours after immersion.

4. There were no differences in size and shape of the caffeine-induced contractures between the muscle immersed in Ringer solution for 24 hours and the control.

5. In the muscle surviving for 24 hours in Ringer solution, the threshold concentration of Ach to produce contractures was about 10 times higher than that in the control.

6. It is concluded that the failure of the E-C coupling processes in the surviving nerve-muscle preparations starts in earlier stage prior to functional disturbances of the end-plate, and remains incomplete even after 24 hours when the end-plate transmission fails completely.

The author is deeply indebted to Professor T. NAGAI and Dr. T. YAmaguchi for their valuable advices and encouragement.

This work was supported in part by a grant in Aid for Fundamental Scientific Research from the Ministry of Education.

\section{REFERENCES}

1) Hodgkin, A. L. and Horowicz, P. The differential action of hypertonic solutions on the twitch and action potential of a muscle fibre. J. Physiol. 136:17p, 1957.

2) Yamaguchi, T., Matsushima, T., Fujino, M. and Nagai, T. The excitation-contraction coupling of the skeletal muscle and the 'glycerol effect'. Jap. J. Physiol. $12: 129,1962$.

3) Bülbring, E. and Lüllmann, H. The effect of metabolic inhibitors on the electrical and mechanical activity of the smooth muscle of the guinea-pig's taenia coli. J. Physiol. $136:$ 310, 1957.

4) Kuschinsky, G., Lüllmann, H. and Muscholl, E. The separation of the stimulation process and contraction of skeletal and heart muscle by $2: 4$-dinitrophenol. Naturwissenschaften $45: 520,1958$.

5) Merton, P. A. Voluntary strength and fatigue. J. Physiol. 123: 553, 1954.

6) Seraidarian, M. W., Abbott, B. C. and Williams, E. B. Studies on the frog's sartorius at different stages of activity. Biochim. Biophys. Acta $46: 355,1961$.

7) Mashima, H., Matsumura, M. and Nakayama, Y. On the coupling relation between action potential and mechanical response during repetitive stimulation in frog sartorius muscle. Jap. J. Physiol. 12: 324, 1962.

8) SANDOW, A. Potentiation of muscular contraction by the nitrate-ion. Science 112 : 647,1950 .

9) Lissák, K., Dempsy, E. W. and Rosenblueth, A. Failure of transmission of motor nerve impulses in course of Wallerian degeneration. Am. J. Physiol. 128: 45, 1939.

10) Kuffler, S.W. Specific excitability of the end-plate region in normal and denervated muscle. J. Neurophysiol. 6: 99, 1943.

11) Birks, R., KAtz, B. and Miledi, R. Physiological and structual changes at the amphibian myoneural junction, in course of the nerve degeneration. J. Physiol. 150: 145, 1960.

12) TASAKI, I. The strength-duration relation of the normal, polarized and narcotized nerve fibre. Am. J. Physiol. $125:$ 367, 1939. 
13) Macfarlene, W. V. and Meares, J.D. Chemical modification of intracellulary recorded after-potentials of frog skeletal muscle. J. Physiol. 142: 78, 1958.

14) St ̈̈мpғLI, R. A new method for measuring membrane potentials with external electrodes. Experimentia 10:508, 1954.

15) Nicholls, J.G. The electrical properties of denervated skeletal muscle. J. Physiol. $131: 1,1956$.

16) Siekevitz, P. On the meaning of intracellular structure for metabolic regulation. Regulation of Cell Metabolism, Ciba Fundation Symposium 17p. 1959.

17) Axelsson, J. and TheslefF, S. A study of supersensitivity in denervated mammalian skeletal muscle. J. Physiol. $147:$ 178, 1959.

18) Elmqvist, D. and Thesleff, S. A study of Acetylcholine induced contractures in denervated mammalian muscle. Acta Pharmacol. et Toxicol. 17: 84, 1960.

19) Gelfan, S. in Fulton, J.F. (Editor) Functional activity of muscle, A Textbook of Physiology 17th ed., 154, 1955. W. B. Saunders Company, Philadelphia. 